\title{
Metal oxide/polyaniline nanocomposites: Cluster size and composition dependent structural and magnetic properties
}

\author{
RAKSHA SHARMA, RAKESH MALIK, SUBHALAKSHMI LAMBA ${ }^{\dagger}$ and S ANNAPOORNI* \\ Department of Physics and Astrophysics, University of Delhi, Delhi 110 007, India \\ ${ }^{\dagger}$ School of Sciences, Indira Gandhi National Open University (IGNOU), New Delhi 110 068, India
}

\begin{abstract}
Nanocomposites of iron oxide with conducting polymer in the form of powders of varying compositions have been studied to understand the effects of particle size, cluster size and magnetic inter-particle interactions. The sizes of the nanoparticles were estimated to be $\sim 10-20 \mathbf{~ n m}$ from the X-ray diffraction (XRD) and the transmission electron micrographs (TEM). XRD shows a single crystalline phase for the $\gamma-\mathrm{Fe}_{2} \mathrm{O}_{3}$. The presence of conducting polymer was confirmed through Fourier transform infrared (FTIR) spectroscopy. The amount of polymer present in the composite, the transition temperature of iron oxide and the thermal stability of polymer was determined through thermogravimetric and differential thermal analysis (TGA-DTA). The room temperature magnetic hysteresis measurements show reduction in saturation magnetization with increasing polymer concentrations. A low value of coercivity was observed for low polymer composites. On increasing the polymer concentration, the coercivity and remanence become negligible indicating a superparamagnetic phase at room temperature. Beyond a certain composition, the system shows paramagnetic behaviour which is also confirmed through zero field cooled-field cooled (ZFC-FC) measurements. We also report preliminary results on the magnetic properties of self standing sheets prepared using $\gamma-\mathrm{Fe}_{2} \mathrm{O}_{3}$ and $\mathrm{NiFe}_{2} \mathrm{O}_{4}$ nanoparticles and conducting polymers.
\end{abstract}

Keywords. Metal oxide/polyaniline nanocomposites; structural properties; magnetic properties.

\section{Introduction}

The diverse properties of magnetic nanoparticle systems have elicited wide interest from the point of view of technological applications. Their properties are known to be strongly dependent on size, anisotropy and inter particle interactions (both exchange and dipolar) (Dormann and Fiorani 1992). Theoretical interest in these systems stems from the complex behaviour arising from the interplay of these factors (Papusoi et al 1999) and the possibility of fine tuning them for wider applicability. Since magnetic nanoparticles are prone to agglomeration, which reduces their usability, they can be dispersed in a non-magnetic matrix to form nanocomposites (Chen et al 1997), to reduce agglomeration. The matrix plays an important role in the relaxation and blocking temperature of magnetic nanoparticles (Sharma et al 2006). We have earlier studied the role of interactions in such nanocomposites (Sharma et al 2005a). In the present investigations, we report the structural and magnetic properties of iron oxide/polyaniline nanocomposite powders prepared by the polymerization over core (POC) method, as a function of composition. An attempt was also made to obtain self standing sheets of polyaniline dispersed with iron oxide and nickel ferrite nanoparticles and hysteresis measure-

*Author for correspondence (aalqudami@physics.du.ac.in) ments were performed on these sheets. We also report the results of Monte Carlo simulations performed on an interacting model for single domain magnetic particles and a comparison is made with experimental results.

\section{Experimental}

Iron oxide nanoparticles were prepared by the sol-gel method using iron nitrate $\left(\mathrm{Fe}\left(\mathrm{NO}_{3}\right)_{3} \cdot 9 \mathrm{H}_{2} \mathrm{O}, 99.99 \% \mathrm{pu}-\right.$ rity, Aldrich $\mathrm{Co}$.) as precursor and 2-methoxy ethanol $\left(\mathrm{C}_{3} \mathrm{H}_{8} \mathrm{O}_{2}, 99.90 \%\right.$ purity $)$ as solvent. A weighed quantity of the precursor was added to 2-methoxy ethanol, this solution was then filtered and heated to $50^{\circ} \mathrm{C}$. The gel obtained was heated in the temperature range $150-200^{\circ} \mathrm{C}$. The powder obtained was ground in a motor and pestle, sonicated in distilled water, centrifuged and dried at $50^{\circ} \mathrm{C}$. The iron oxide/polyaniline nanocomposites in various compositions were prepared by polymerizing aniline monomers over iron oxide particles through chemical oxidation (polymerization over core process, POC) (Sharma et al 2005b). The compositions of iron oxide: aniline monomers prepared were $1: 0 \cdot 1,1: 0 \cdot 2,1: 0 \cdot 4$, $1: 0 \cdot 8,1: 1,1: 2$ and $1: 3$.

In an attempt to better understand the role of interparticle interactions, the nanoparticles were dispersed in polymer matrix in the form of self standing sheets. Self standing sheets of nickel ferrite nanoparticles in a poly- 
mer matrix were also prepared using nickel ferrite particles of $98+\%$ purity purchased from Aldrich Company. For this purpose polyaniline was prepared by chemical oxidation of aniline monomer with ammonium persulphate as oxidant in an acidic medium (here $\mathrm{HCl}$ was used) at $0-5^{\circ} \mathrm{C}$. A dark green powder was obtained which is an emeraldine salt. This powder was stirred continuously in ammonia solution for $24 \mathrm{~h}$ to obtain the emeraldine base powder which is highly processible. This powder was dissolved in $N$-methyl pyrrolidone solution and filtered. The metal oxide nanoparticles in weighed quantity were dispersed in the above solution and sonicated. Free standing iron oxide/PANI and nickel ferrite/PANI nanocomposite films (of thickness $\sim 100 \mu \mathrm{m}$ and $10 \times 10 \mathrm{~cm}$ ) were prepared by heating the above solutions in a convection oven at $60-70^{\circ} \mathrm{C}$ for $1-3 \mathrm{~h}$.

The composites obtained by these two methods were subjected to various structural and magnetic measurements. The phase present and average grain size was determined by XRD performed using a Rigaku Rotaflex diffractometer. Scanning electron microscopy (SEM) was used to study the morphology of the film using JEOL JSM 840 SEM. To confirm the presence of PANI, FTIR measurements were performed on the composites using $\mathrm{KBr}$ pellets by a Perkin-Elmer FTIR spectrum 2000 spectrometer. The room temperature hysteresis loops were measured using a vibrating sample magnetometer (VSM-5, TOEI Industry Co. Ltd, Tokyo, Japan). A Quantum Design MPMS-5S

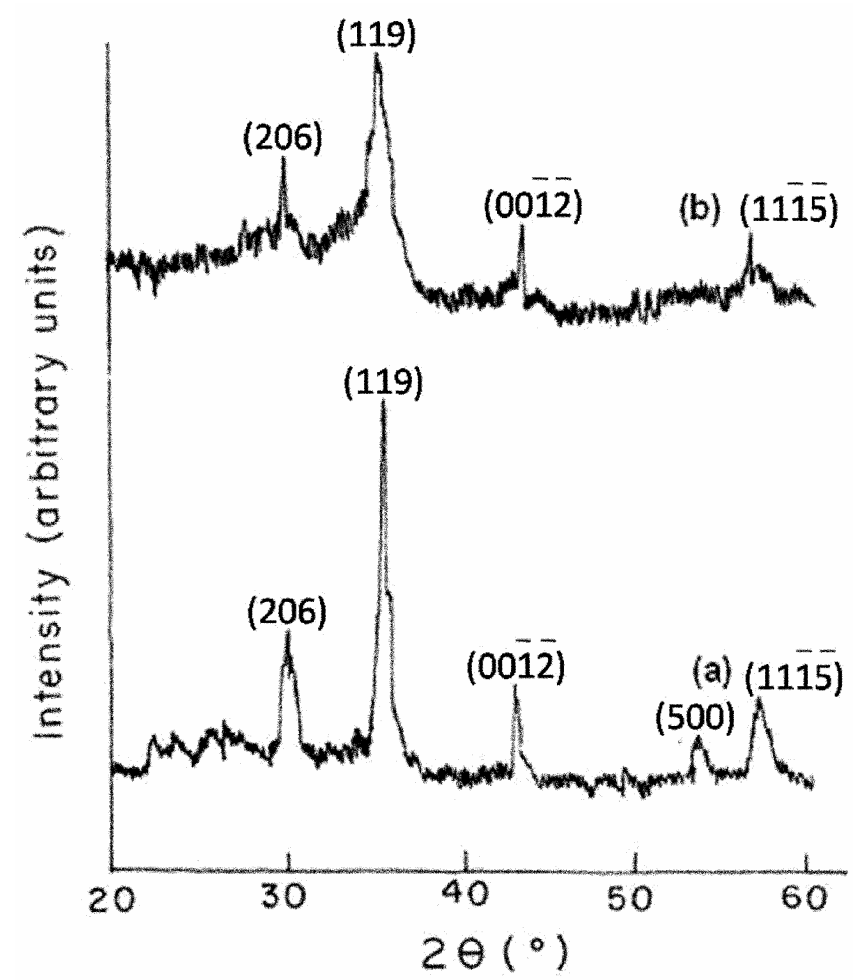

Figure 1. XRD of (a) iron oxide nanoparticles and (b) iron oxide/PANI ( $1: 0.4)$ composite.
SQUID magnetometer was used for ZFC-FC measurements.

\section{Results and discussion}

\subsection{Structural characterization}

XRD shows a single phase of $\gamma-\mathrm{Fe}_{2} \mathrm{O}_{3}$. The lattice constants calculated were $a=8.34 \AA$ and $c=25.02 \AA$ for maghemite and $a=8.34 \AA$ for nickel ferrite nanoparticles, respectively which compares well with the values reported in literature (Craik and Tebble 1965). The same phase of the metal oxides were retained in their respective nanocomposites with PANI, although a broadening of the peaks is observed, indicating a reduction in grain size (figure 1). The TEM shows agglomerated clusters of iron oxide. Agglomeration is reduced by a great amount on making composites. Chains of particles and intra chain structures were observed for particular compositions of iron oxide/PANI composites (Sharma et al 2005b). The SEM studies of the self standing sheets show the presence of small clusters in the composite sheets (figure 2), while fibrous structures are observed in the PANI sheets (inset of figure 2). The peaks in the FTIR spectra at 990 and $1022 \mathrm{~cm}^{-1}$ are identified as $=\mathrm{C}-\mathrm{H}$ bending, at 1477 and $1561 \mathrm{~cm}^{-1}$ for $\mathrm{N}-\mathrm{H}$ bonds, and at 690 and $804 \mathrm{~cm}^{-1}$ as the $\mathrm{C}-\mathrm{H}$ bending, respectively confirming the polymerization of aniline monomers. The peaks observed between 400 and $700 \mathrm{~cm}^{-1}$ correspond to $\mathrm{Fe}-\mathrm{O}$ bonding of iron oxide. The differential thermal analysis shows the phase change of iron oxide (from maghemite to hematite) at $669 \mathrm{~K}$. The transition temperature $\left(T_{\mathrm{M}}\right)$ was found to be reduced from 669 to $623 \mathrm{~K}$ on making its composites. Self standing composite sheets do not show much variation in $T_{\mathrm{M}}$. The polymer degradation temperature was found to

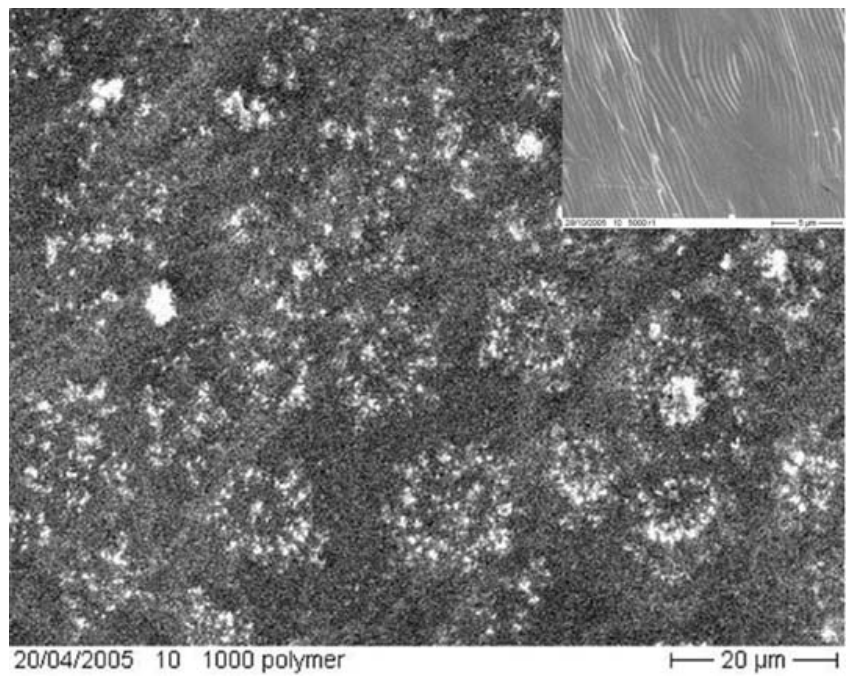

Figure 2. SEM of iron oxide/PANI self standing sheet (Inset shows the PANI sheet). 
be 583 and $643 \mathrm{~K}$ for iron oxide/PANI composite powders and sheets, respectively.

\subsection{Effect of composition on coercivity}

The magnetic properties viz. coercivity, remanence and saturation magnetization, show a considerable variation with change in composition. The room temperature hysteresis shows a decrease in saturation magnetization $\left(M_{\mathrm{S}}\right)$ with increase in polymer concentration (figure 3 ), and beyond a concentration of $1: 2$ it shows a paramagnetic behaviour (figure 4). The paramagnetic behaviour was also confirmed by $\mathrm{ZFC}-\mathrm{FC}$ measurements (inset of figure 4). Figure 5 shows the variation of coercivity $\left(H_{\mathrm{C}}\right)$ as a

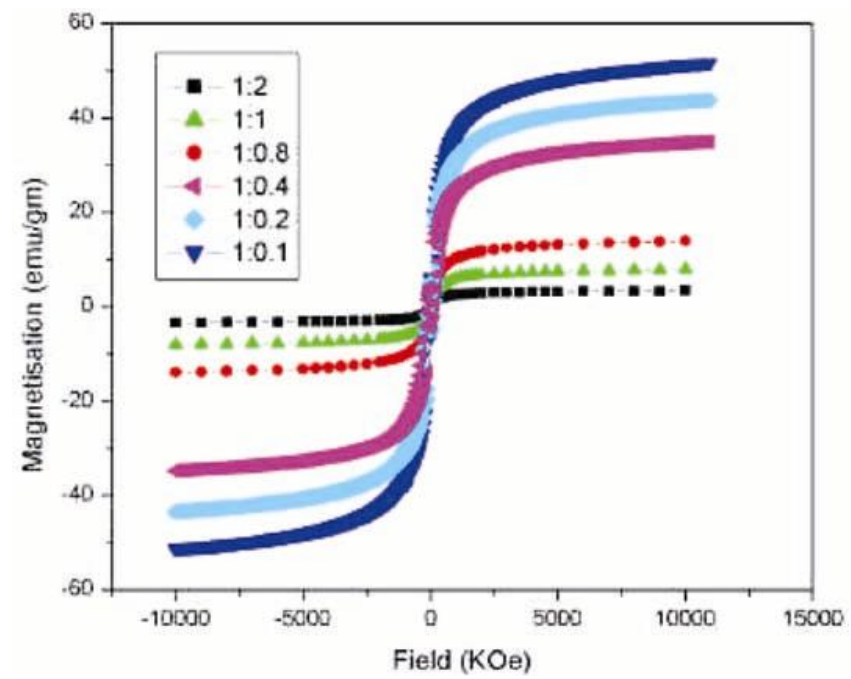

Figure 3. Room temperature hysteresis for iron oxide/PANI composites of various compositions

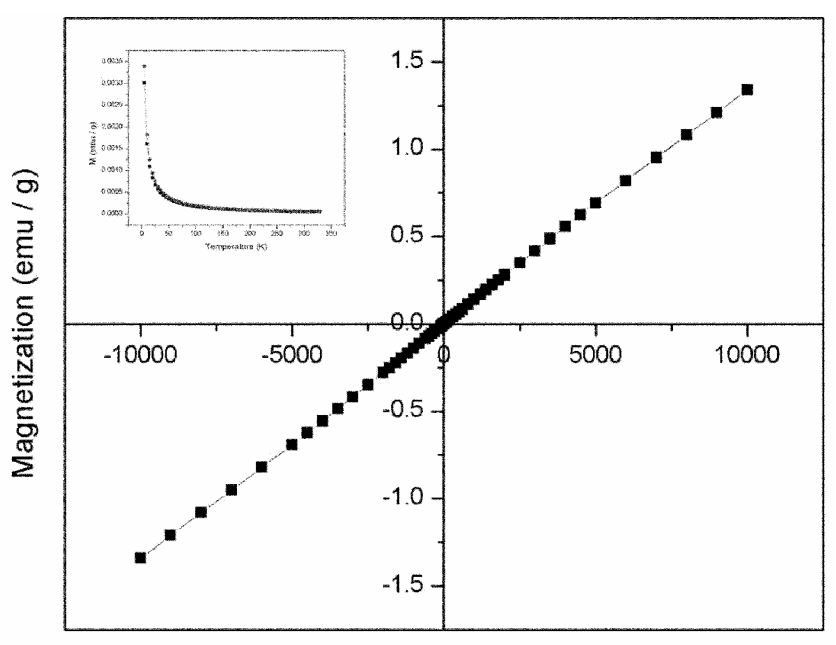

Field $(\mathrm{Oe})$

Figure 4. Room temperature hysteresis for iron oxide/PANI ( $1: 3$ ) composite (Inset shows ZFC-FC measurement) function of composition. A $H_{\mathrm{C}}$ of $\sim 155-160$ Oe and remanence $\left(M_{\mathrm{R}}\right)$ of $\sim 6-8 \mathrm{emu} / \mathrm{g}$ was observed for low polymer concentration. With increasing polymer composition, the $H_{\mathrm{C}}$ and $M_{\mathrm{R}}$ decreases to $1.2 \mathrm{Oe}$ and $0.03 \mathrm{emu} / \mathrm{g}$, respectively. Thus the system remains ferromagnetic for low polymer concentration and reaches superparamagnetic limit for higher polymer concentration. With decreasing particle concentration, both the exchange and dipolar interactions are weakened and the system shows progressively lower coercivity and magnetization.

The hysteresis measurements at $5 \mathrm{~K}$ for self standing sheets of iron oxide: PANI $(1: 2)$ and nickel ferrite : PANI (1:5) show $M_{\mathrm{S}}, H_{\mathrm{c}}$ and $M_{\mathrm{r}}$ of $14.1 \mathrm{emu} / \mathrm{g}, 430 \mathrm{Oe}$ and $6.06 \mathrm{emu} / \mathrm{g}$ and $7.95 \mathrm{emu} / \mathrm{g}, 584 \mathrm{Oe}$ and $3.11 \mathrm{emu} / \mathrm{g}$, respectively (figure 6). Further investigations of the self-

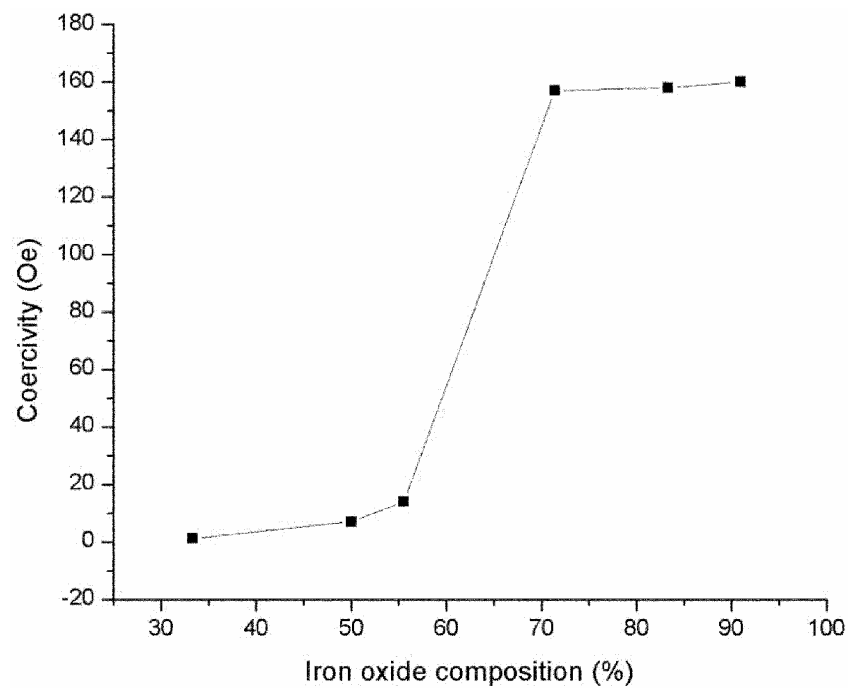

Figure 5. Coercivity as a function of iron oxide in the composite.

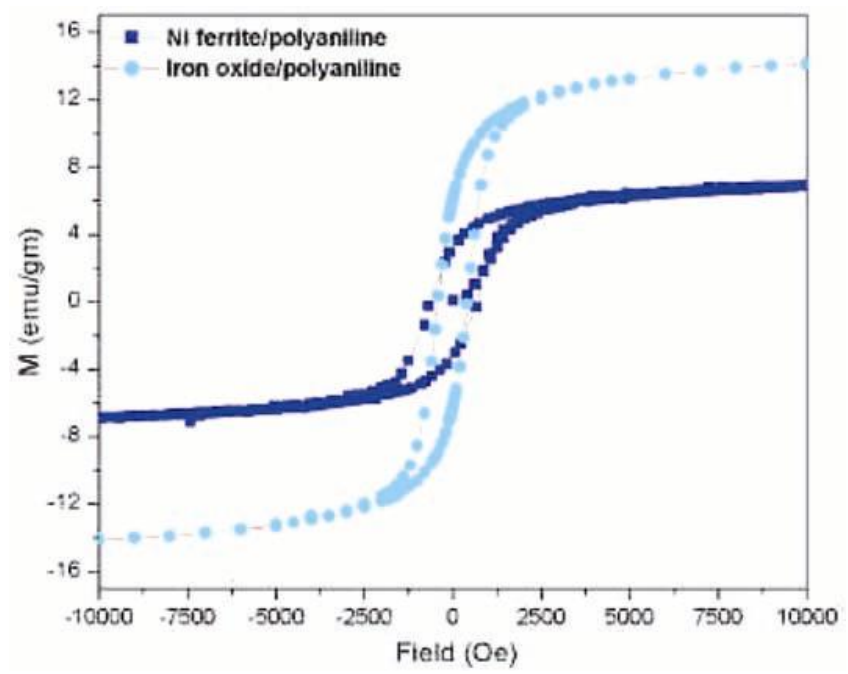

Figure 6. Hysteresis measurements of iron oxide/PANI and nickel ferrite/PANI self standing sheets at $5 \mathrm{~K}$ 
standing composites sheets are being carried out to understand the role of matrix on magnetic properties.

\section{Numerical studies}

An array of interacting, single domain magnetic particles driven by a magnetic field of strength, $B$, is described by the following Hamiltonian (Lamba and Annapoorni 2004)

$$
\begin{aligned}
H & =-K \sum_{i} V_{i} \frac{\left(\vec{\mu}_{i} \cdot \vec{n}_{i}\right)^{2}}{\left|\vec{\mu}_{i}\right|^{2}}-\sum_{(i \neq j)} J \vec{\mu}_{i} \cdot \vec{\mu}_{j} \\
& -\mu_{0} \sum_{(i \neq j)} \frac{3\left(\vec{\mu}_{i} \cdot \vec{e}_{i j}\right)\left(\vec{\mu}_{j} \cdot \vec{e}_{i j}\right)-\vec{\mu}_{i} \cdot \vec{\mu}_{j}}{r_{i j}^{3}}-\mu_{0} \sum_{j} \vec{B} \cdot \vec{\mu}_{j},
\end{aligned}
$$

where $\vec{\mu}_{i}$ is the magnetic moment vector for the $i$ th particle and $V_{i}$ its volume, $K$ the anisotropy energy per unit volume and $J$ the strength of the exchange interaction between particles. The properties of such an array depends upon the material parameters like the size and shape of the particles and the anisotropy energy, as also on the interactions present in the system, which are both the long range dipolar interactions as well as the short range exchange interactions. The interplay of interactions becomes especially important when one is studying the composition dependent properties of magnetic nanocomposite systems. We have studied earlier that interactions, in particular, the inter-particle exchange interactions in composites can account for the high blocking temperatures (close to room temperature) observed in these systems (Sharma et al 2005b). With increasing dilution of the magnetic component in the composite, inter-particle distances increase and both the exchange and dipolar interactions become weaker. This destroys the long range magnetic order in the composite and the system eventually becomes paramagnetic, as is reported in this paper, for the iron oxide-PANI composite powders with a composition of $1: 3$ (figure 4). The properties of iron oxidePANI composite powders have been analysed numerically in an earlier study (Sharma et al 2005b) and the anisotropy for this material was estimated to be $\sim 0.75 \times$ $10^{5} \mathrm{~J} / \mathrm{m}^{3}$. In our present study, we explain through our simulation the unusual behaviour observed in the variation of the room temperature coercivity with composition for the iron oxide-PANI composite powders (figure 5) using this value of the anisotropy. We also estimate afresh the anisotropy energy for the two composite sheets reported in this paper using the hysteresis data. The details of the system and Monte Carlo simulation are the same as reported in Sharma et al (2005b).

In figure 7 we plot the variation of the coercivity of the powder nanocomposite of iron oxide with the concentration of iron oxide in the composite. The concentration is calculated in units of the number of iron oxide particles per unit volume of the simulation cell. The average particle size is the Gaussian distribution of particle diameter of $15 \mathrm{~nm}$. At both the low and high concentrations, the coercivity remains almost constant-as is also seen in the experimental curve (figure 5). Our understanding of this behaviour is as follows: at low concentrations the inter particle separations are so large that both dipolar and exchange interactions are minimal and the magnetic properties of nanocomposites are dominated by the effects of anisotropy. Hence, at these concentrations the room temperature coercivity is very low and remains constant

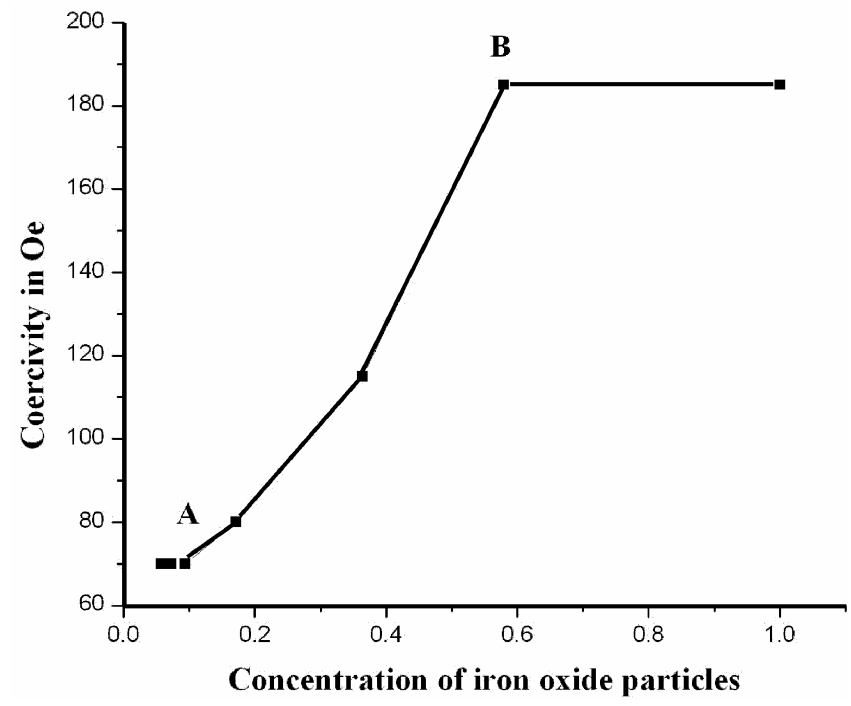

Figure 7. Variation of the coercivity (calculated theoretically) of the iron oxide/PANI nanocomposite with iron oxide concentration.

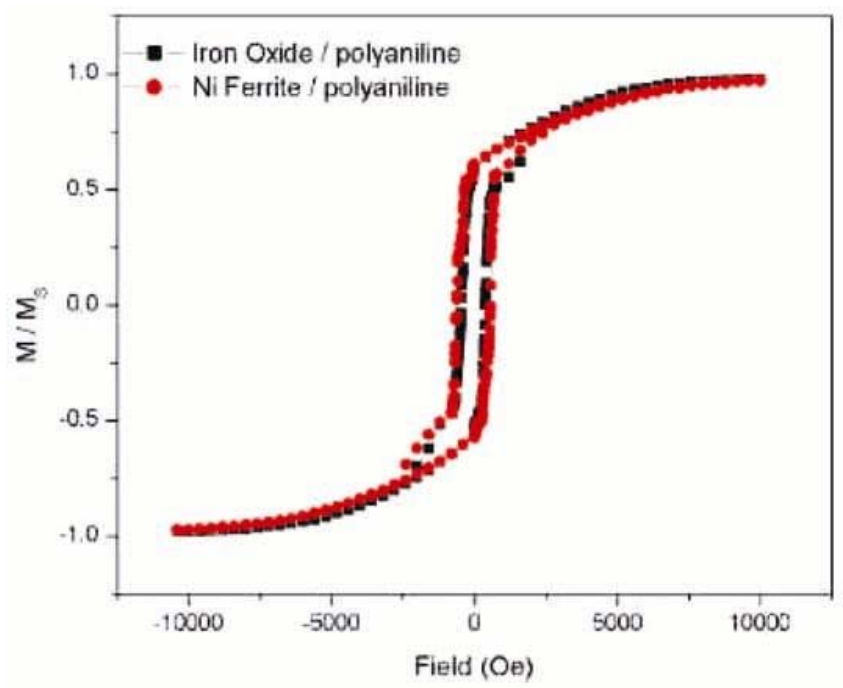

Figure 8. Simulated hysteresis curves at $5 \mathrm{~K}$, for the two types of composite sheets, nickel ferrite/PANI (curve a) and iron oxide/PANI (curve b). 
up to those concentrations for which the average anisotropy field in the system is larger than the interaction field, which is the point $\mathrm{A}$ in figure 7. For concentrations beyond $\mathrm{A}$, when the interaction fields become comparable to the anisotropy field, the coercivity is decided by an interplay of anisotropy, dipolar and exchange interactions. With decreasing inter particle distances, the effect of interactions gradually increases, in particular interparticle exchange interactions become important, which is why the coercivity of the system gradually increases up to the point $B$ in figure 7 . Beyond $B$ the exchange effects totally dominate the anisotropy effects in the system and once again further changes in concentrations do not result in much change in the coercivity.

In figure 8 , we plot the simulated hysteresis at $5 \mathrm{~K}$, for the two types of composite sheets, nickel ferrite-PANI (curve a) and iron oxide-PANI (curve b) and estimate the anisotropy by matching the simulated hysteresis with the experiments. The simulated coercivity at these temperatures is 595 Oe and 420 Oe, respectively for the two systems. The average particle sizes for the two systems are 10 and $15 \mathrm{~nm}$, respectively. The values of the anisotropy energies at which we are best able to fit the experimental data is $1.25 \times 10^{5} \mathrm{~J} / \mathrm{m}^{3}$ for the nickel ferrite composite sheet and $1.05 \times 10^{5} \mathrm{~J} / \mathrm{m}^{3}$ for the iron oxide composite sheet.

\section{Conclusions}

Our experiments indicate that for fairly low concentrations of polyaniline in nanocomposites the ferromagnetic phase is retained. However, for high polymer concentrations the very low value of coercivity suggests that the system is close to superparamagnetic behaviour. Beyond a certain concentration of polymer the system shows paramagnetic behaviour. Theoretical calculations show that the anisotropy is relatively higher for self standing sheets as compared to the composites in powder form, indicating that the ferromagnetic phase will be retained up to higher temperatures for these sheets.

\section{Acknowledgements}

(RS) and (RM) wish to acknowledge the Council of Scientific and Industrial Research and the University Grants Commission, India, respectively for financial support. The authors wish to acknowledge DST, India, for financial assistance through their project (SR/S5/NM-52/2002) from their Nano Science and Technology Initiative Program. They also acknowledge the help extended by Dr N C Mehra and Dr S K Shukla, USIC, Delhi University, New Delhi. We are thankful to Dr Parmanand and Dr Kotnala for help extended in magnetic measurements.

\section{References}

Chen L, Yang W J and Yang C Z 1997 J. Mater. Sci. 323571

Craik D J and Tebble R S 1965 Ferromagnetism and ferromagnetic domains (ed.) E P Wohlfarth (Amsterdam: North Holland) 4 p. 95

Dormann J H and Fiorani D 1992 Magnetic properties of fine particles (Amsterdam: North Holland)

Lamba S and Annapoorni S 2004 Euro. Phys. J. B39 19

Papusoi Jr C 1999 J. Magn. Magn. Mater. 195708

Sharma R, Lamba S and Annapoorni S 2005a J. Phys. D. Appl. Phys. 383354

Sharma R, Lamba S, Annapoorni S, Sharma P and Inoue A 2005b J. Appl. Phys. 97014311

Sharma R, Suri K, Tandon R P, Annapoorni S, Lamba S and Kumaraswami B V 2006 J. Appl. Phys. 99024311 